\title{
Flavonoids: A vital resource in healthcare and medicine
}

\begin{abstract}
There are many biological resources in nature that are essential in relation to health care. Due to their flexible health benefits documented in various epidemiological studies, there has been increasing interest in research on flavonoids from plant sources. Since flavonoids are directly related to human dietary ingredients and health, structure and function relationships need to be evaluated. Flavonoids bioavailability, metabolism, and biological activity depends on the composition, total number of hydroxyl groups, and functional group substitution regarding their nuclear structure. Fruits and vegetables, together with tea and wine, are the primary dietary sources of flavonoids for humans. This review article primarily focusses on demarcating the importance of flavonoids which shows many pharmacological actions by acting as anti-cancer, anti-oxidant, anti-hypertensive and anti-cholinesterase agents. These flavonoids had shown positive results in treatment of pregnancy induced disorders. We also tried to discuss few studies which proves the efficiency of flavonoids to treat COVID-19. They had also been proved to show greater potential in treatment of Neurodegenerative diseases. Nanomedicine also has a greater part to play in near future as it helps flavonoids show effective targeted drug delivery.
\end{abstract}

Keywords: nanomedicine, pregnancy induced hypertension, anti-cancer, bioavailability, anti-cholinesterase activity

\section{Introduction}

Flavonoids are polyphenolic phytochemicals present in a variety of food products and beverages classified into six groups of different chemical structures and physiological properties. Evidence from various epidemiological studies support the notion that chronic flavonoid-rich diets may be associated with a reduced risk of certain chronic diseases, including cardiovascular, neurodegenerative and select cancers. In this context, particular attention has been paid to two subclasses of flavonoids and flavanols.
Volume 8 Issue 2 - 2020

\section{Sumanta Mondal, Syed Tazib Rahaman}

Associate Professor, GITAM Institute of Pharmacy, India

Correspondence: Syed Tazib Rahaman, GITAM Institute of Pharmacy, GITAM (Deemed to be University),Visakhapatnam, Andhra Pradesh, India,Tel 0770214986।,

Email tazib.research@gmail.com

Received: April 06, 2020 | Published: April 24, 2020

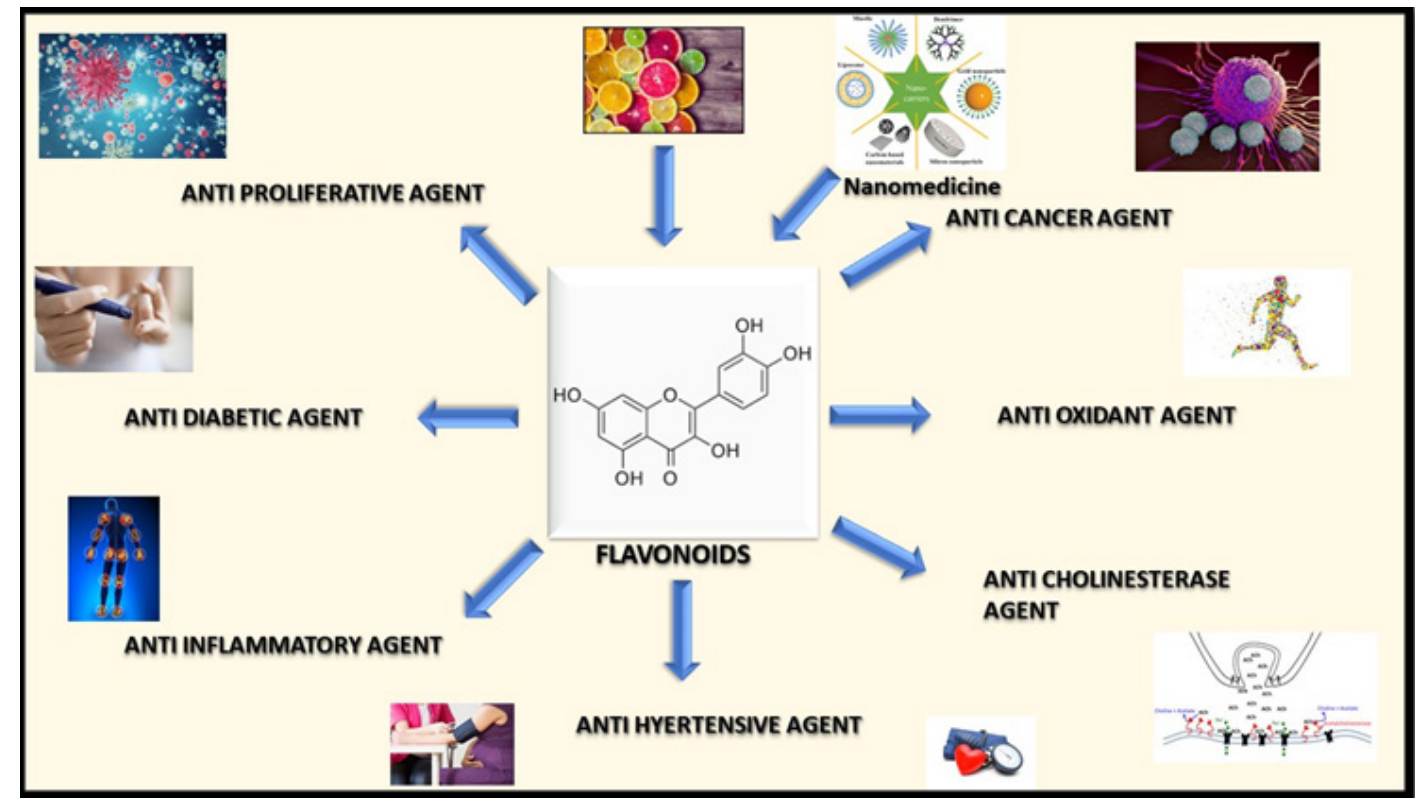

Figure I Graphical abstract.

Flavonoids consist of a large group of polyphenolic compounds with a structure of benzo- $\pi$-pyrone and are ubiquitous in plants. Phenyl propanoid pathway synthesizes them. Studies available tend to show that secondary phenolic metabolites like flavonoids are responsible for the variety of pharmacological activities. Flavonoids are phenolic hydroxylated substances and in response to microbial infection are known to be synthesized by plants. Our activities depend on the structure. ${ }^{2}$ Flavonoids chemical composition depends on their structural group, degree of hydroxylation, other substitutes and conjugations, and degree of polymerization (Figure 1). 
The potential health benefits arising from the antioxidant activities of these polyphenolic compounds have sparked recent interest in these substances. By scavenging free radicals and/or chelating metal ions, active hydroxyl groups in flavonoids mediate their antioxidant effects. ${ }^{3-5}$ Metal chelation may be crucial in preventing radical generation that damages target biomolecules. Because of their high antioxidant potential both in vivo and in vitro systems, flavonoids are thought to have health-promoting properties as a dietary element. The ability of flavonoids to activate human enzyme defence systems. The number of studies indicated protective effects against many infections. ${ }^{6}$

\section{Chemistry}

Flavonoids are a class of natural compounds that are present in plants with variable phenolic structures. A new material from oranges was isolated in 1930. It was thought to be a member of a new class of vitamins at that time and was referred to as vitamin P. It became evident later that this material was a flavonoid (rutin) and more than 4000 varieties of flavonoids have been described so far. ${ }^{7}$ Chemically flavonoids are based on a skeleton of fifteen carbon composed of two benzene rings connected by a heterocyclic pyrane chain. The different classes of flavonoids vary in the degree of oxidation and the substitution pattern of the $\mathrm{C}$ ring, while individual compounds differ in the pattern of substitution of the A and B rings. ${ }^{8}$

Flavonoids are known as aglycones, glycosides and derivatives that are methylated. The basic structure of flavonoids is aglycone. Either a $\alpha$-pyrone or its dihydroderivative is a six-member ring condensed with the benzene chain. The benzenoid substituent's position separates the group of flavonoids into flavonoids and isoflavonoids. At the 3-position, flavonols differ from flavanones by hydroxyl group and a double bond between $\mathrm{C} 2$ and $\mathrm{C} 3$. In positions 3, 5, 7, 2, 3', 4' and 5', flavonoids are often hydroxylated. The alcohol group's methyl ethers and acetyl esters are known to occur in nature (Figure 2). ${ }^{9-11}$

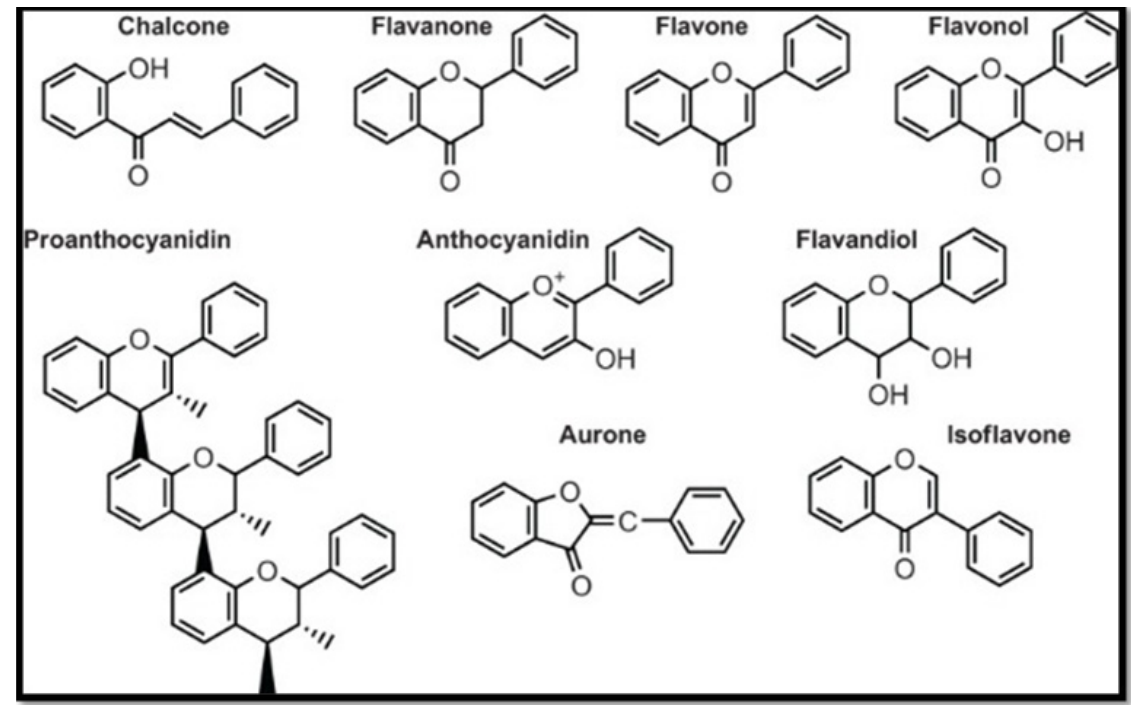

Figure 2 Chemical structures of various flavonoids.

\section{Identification test for flavonoids}

All flavonoids give fluorescence on UV at 254 and $365 \mathrm{~nm}$. According to the structural types of flavonoids show dark yellow, green or blue fluorescence, which is intensified and changed by the use of various spray reagents. Such as

\section{Spray reagents}

Fast blue salt reagent (FBS): $0.5 \mathrm{~g}$ fast blue salt $\mathrm{B}$ is dissolved Table I Identification tests of flavonoids in $100 \mathrm{ml}$ water (Fast blue $\mathrm{B}=3,3$ '- dimethoxybiphenyl-4, 4' bisdiazonium-dichloride) it is generally used for detection of Phenolic compounds.

Natural products-polyethylene glycol reagent (NP/PEG-reagent or NEU-reagent): It is used for diction of flavonoids and intense fluorescence is produced in UV - $365 \mathrm{~nm}$. The fluorescence behaviour is structure dependent (Table1).

\begin{tabular}{lll}
\hline Class or structural types of flavonoids & Name of the flavonoids & Colour of the fluorescence \\
\hline \multirow{2}{*}{ Flavonols } & Quercetin, Myricetin and their glycosides & Orange-yellow \\
& Kaempferol and their glycosides & Yellow-green \\
& Luteolin and their glycosides & Orange \\
Flavonols & Apigenin and their glycosides & Yellow-green \\
\hline
\end{tabular}




\section{General chemical test for flavonoids}

Shinoda tests (magnesium $+\mathrm{HCl}$ ): To the aqueous or alcoholic solution of the extract, add a piece of magnesium ribbon and few drops of concentrated hydrochloric acid. A pink or magenta colour develops which indicates presence of flavonoids.

With $\mathrm{NaOH}$ : The extract dissolved in water, filtrate treated with sodium hydroxide, a yellow colour is observed if flavonoids are present.

With $\mathrm{H}_{2} \mathrm{SO}_{4}$ : A drop of concentrated sulphuric acid when added to the above, the yellow colour disappears.

Neutral lead acetate test: it gives orange red precipitate with neutral lead acetate $(10 \%$ lead acetate in ethanol), confirmed for flavonoids.

Ferric chloride test: it gives Green colouration for flavonoids.

Lieberman Burchard reagent: To about $2 \mathrm{ml}$ of a solution extract in chloroform in a dry test tube, add $2 \mathrm{ml}$ of acetic anhydride and 2-3 drops of concentrated sulphuric acid. Mix and stand for a few minutes, Pink colours develop if flavonoids are present.

\section{Structure-activity relationship of flavonoids}

The antioxidant activities of flavonoids depend on conjugated hydroxyl groups of the $\mathrm{B}$ and $\mathrm{C}$ ring. By using the following descriptors: $\Delta \mathrm{H}_{\mathrm{f}}$ (the difference in heat of formation between flavonoids and their free radicals resulted after hydrogen atom donation) and HBC (the number of conjugated hydroxyl groups of the $\mathrm{B}$ and $\mathrm{C}$ ring).Hydrogen-donation was thought to be the main mechanism of action of phenolic antioxidants and the difference in heat of formation between the phenolic antioxidant and its free radical produced after $\mathrm{H}$-abstraction $\left(\Delta \mathrm{H}_{\mathrm{f}}\right)$, appeared to be a good index for measuring the scavenging activity of antioxidants (Figure 3 ).

The effectiveness of $\Delta \mathrm{H}_{\mathrm{f}}$ can be understood easily, as it represents the strength of the $\mathrm{O}-\mathrm{H}$ bond. The lower strength of the $\mathrm{O}-\mathrm{H}$ bond corresponds to a higher scavenging activity. Chemical structures, descriptors and antioxidant activity of some flavones and flavonols.

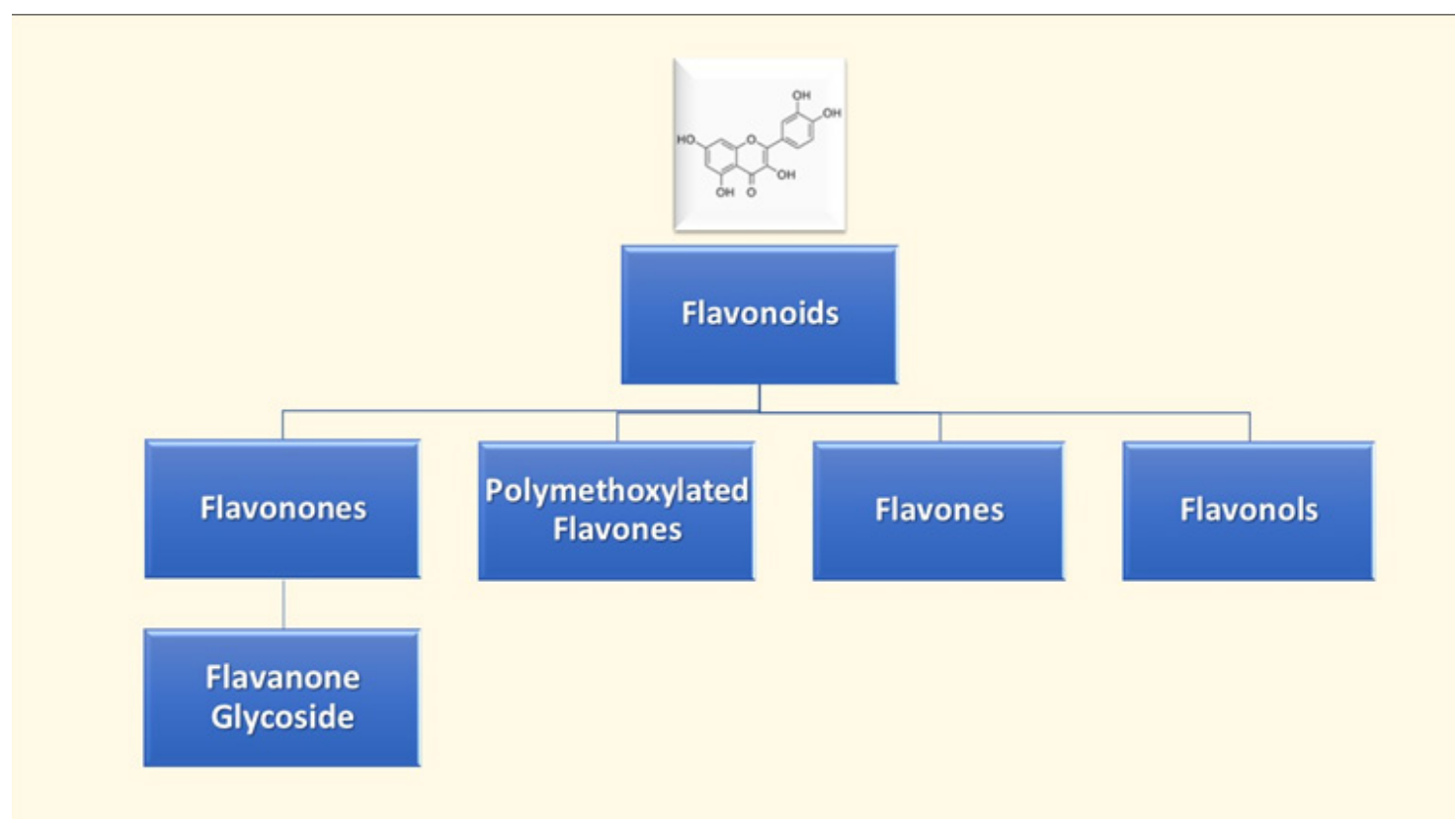

Figure 3 Classification of flavonoids.

By using $\mathrm{HBC}$ and $\Delta \mathrm{H}_{\mathrm{f}}$, the results of this study could be successfully explained as follows:

Flavonoids with $\mathrm{HBC}$ of 0 , such as chrysin failed to show any antioxidant activity because they could not easily donate hydrogen atoms. Flavonoids with $\mathrm{HBC}$ of 1 , such as apigenin showed the poor antioxidant activity, with PFs of 0.99 . Flavonoids with $\mathrm{HBC}$ of 2 , such as kaempferol showed strong antioxidant activity. The PF value of kaempferol is 2.49 .

Flavonoids with $\mathrm{HBC}$ of 3 , such as quercetin showed the strongest antioxidant activity, with $P F$ values of 11.50 . The $\Delta H_{f}$ value of quercetin $(20.84 \mathrm{kcal} / \mathrm{mol})$ was less than that of kaempferol $(21.29$ $\mathrm{kcal} / \mathrm{mol})$, apigenin $(21.29 \mathrm{kcal} / \mathrm{mol})$ and chrysin $(30.92 \mathrm{kcal} / \mathrm{mol})$ which meant the capacity of $\mathrm{H}$-donating of quercetin was stronger than that of chrysin, apigenin and kaempferol. As a result, the antioxidant performance of quercetin was superior.

\section{Structure-activity relationship of flavonoids on their anti-Escherichia coli activity and inhibition of DNA Gyrase}

Flavonoids are potential sources of natural preservatives. Flavonoid inhibitory activities against DNA gyrase from E. coli were estimated by DNA supercoiling. Kaempferol exhibited the greatest antibacterial activity [minimal inhibitory concentration $(\mathrm{MIC})=25 \mu \mathrm{g} /$ $\mathrm{mL}]$, Nobiletin showed the lowest activity $(\mathrm{MIC}=177 \mu \mathrm{g} / \mathrm{mL})$. The structure-activity relationship analysis suggests that, for a good inhibitory effect, the hydroxyl group substitution at $\mathrm{C} 5$ in the A ring and $\mathrm{C}^{\prime}$ ' in the $\mathrm{B}$ ring and the methoxyl group substitution at $\mathrm{C} 3$ and $\mathrm{C} 8$ in the A ring are essential. The presence of the hydroxyl group at $\mathrm{C} 6$ in the $\mathrm{A}$ ring, $\mathrm{C}^{\prime}$ and $\mathrm{C}^{\prime}$ in the $\mathrm{B}$ ring, and $\mathrm{C} 3$ in the $\mathrm{C}$ ring and the methoxyl group at $\mathrm{C}^{\prime}$ in the $\mathrm{B}$ ring greatly reduced inhibition of bacteria. 


\section{Pharmacological activities of flavonoids used during pregnancy}

In foods and drinks such as onions, leeks, broccoli, cherry tomatoes, red wine and tea, flavonols are found. Rutin (quercetin-3rhamnosyl glucoside) is a glycoside of flavonol consisting of quercetin and rutinous disaccharide. ${ }^{12}$

Rutin was thought to have vasoprotective and anticarcinogenic activities. For teas, red grapes, red wines, apples and cocoa-based products, flavanols or flavan-3-ols, including epicatechin and catechin monomers, are widely consumed. Similar to flavonols, vasoprotective properties including anti-inflammatory and vasodilatory activities have been identified in the flavanols. ${ }^{13}$
The results of epidemiological and intervention studies have been largely positive in terms of the putative health benefits of flavanols and flavonols. While there is considerable interest in the potential health benefits of flavonoid intake, there has not been extensive study of the potential adverse effects of consuming very large amounts of these phytochemicals. ${ }^{14,15}$ Many flavonoids are available as dietary supplements for purchase, and it is feasible to achieve levels of intake that significantly exceed what typical diets provide. Because these substances can have pleiotropic effects and many cellular functions, including gene expression and cell signalling, have been shown to be modulated (Figure 4).

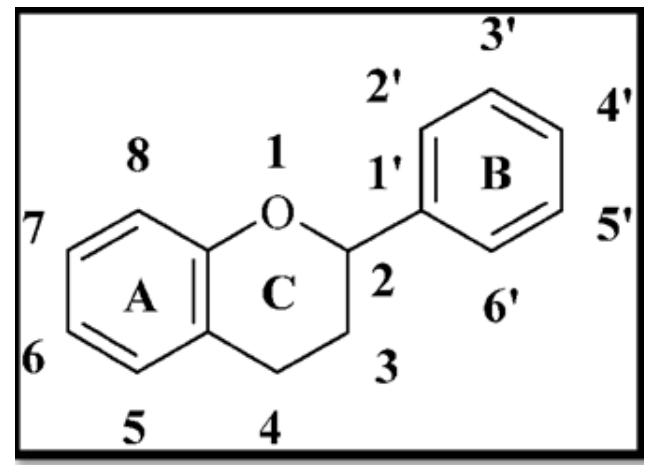

Figure 4 General structure of flavonoids.

The existing literature on the physiological implications of excessive and chronic consumption is minimal, particularly in the context of these nutrients ' pregnancy and developmental toxicity. Current literature suggests that if the intake of all flavonoids during pregnancy is beneficial or harmful to developmental processes, there is uncertainty (Table 2). ${ }^{16}$ Some of these findings come from studies of impaired fetal development (heart, genitourinary and limb abnormalities), impaired absorption and other nutrient metabolism, and chronic lasting effects such as increased risk of early disease onset (e.g. acute childhood leukemia).

Table 2 Recently discovered and most commonly used flavonoid

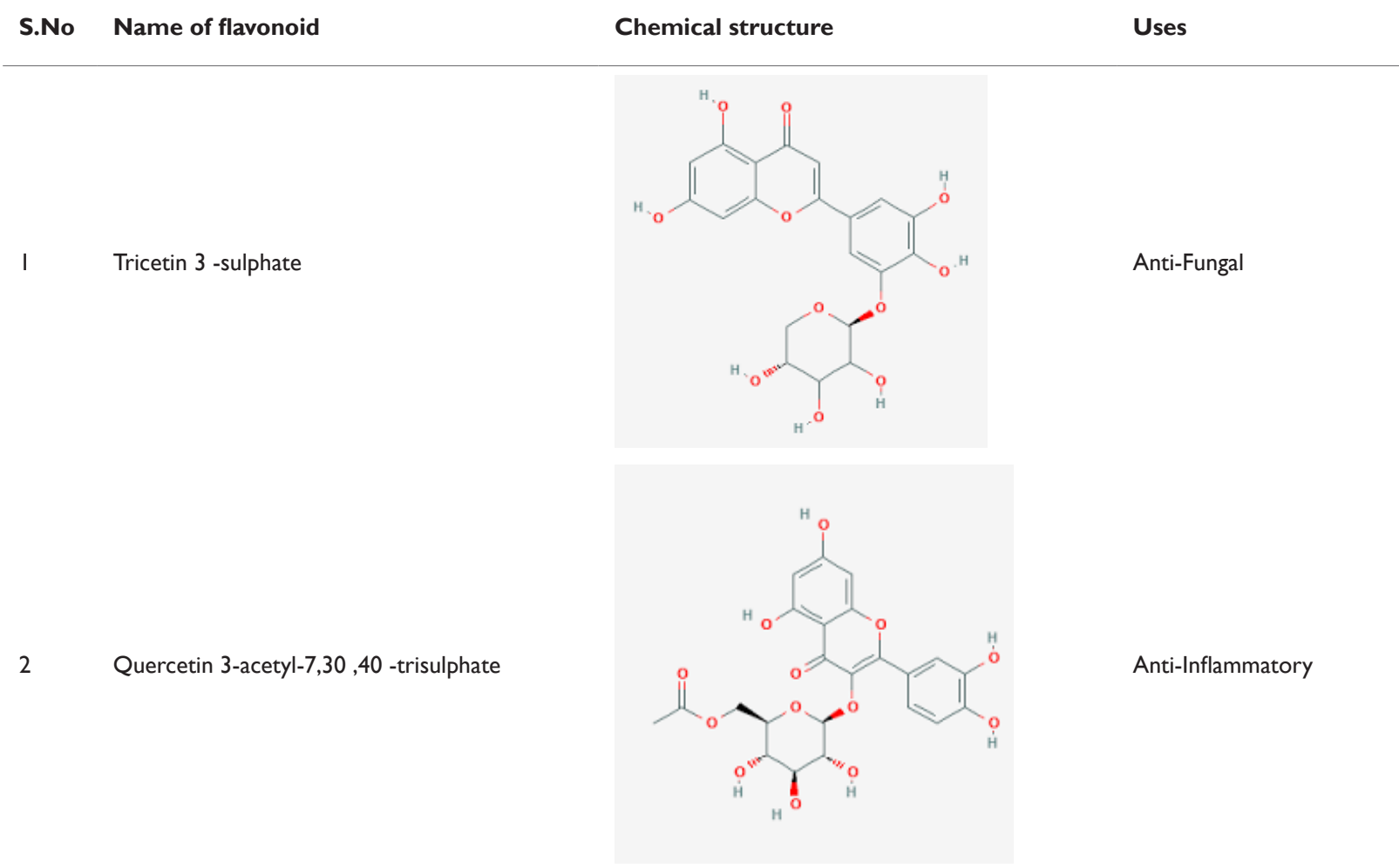


Table Continued...

\section{S.No Name of flavonoid}

Vitexina 7-sulphate

$4 \quad$ Procyanidin

5 Theaflavins

$6 \quad$ Kaempferol

7 Taxifolin
Chemical structure<smiles>[2H][C@@]1(c2c(O)cc(O)c3c(=O)cc(-c4ccc(O)cc4)oc23)O[C@H](CO)[C@@H](O)[C@H](O)[C@H]1O</smiles><smiles>Oc1cc(O)c2c(c1)O[C@H](c1ccc(O)c(O)c1)[C@H](O)[C@H]2c1c(O)cc(O)c2c1O[C@H](c1ccc(O)c(O)c1)[C@H](O)C2</smiles><smiles>O=c1cc(C2Oc3cc(O)cc(O)c3CC2O)cc2c(C3Oc4cc(O)cc(O)c4CC3O)cc(O)c(O)c2c1O</smiles><smiles>O=c1c(O)c(-c2ccc(O)cc2)oc2cc(O)cc(O)c12</smiles>

Anti-oxidan<smiles>O=C1c2c(O)cc(O)cc2O[C@H](c2ccc(O)c(O)c2)[C@@H]1O</smiles>

Anti-Hypotensive

Anti-Diabetic

Anti-Hyperlipidemic 
While the above research supplemented diets with flavonoidrich foods or flavonoid preparations, the effects of chronic intake of different flavonoids during pregnancy have been examined in few studies. In a study, a hypothesis briefed that, during pregnancy and lactation, excessive intake of high amounts of three flavonoids (epicatechin, catechin, and rutin) would result in adverse fetal and neonatal development in a mouse model. ${ }^{17}$

\section{Diabetes mellitus}

Epidemiological studies conducted in the United States and Europe suggest a protective effect of dietary flavonoids for the development of Diabetes Mellitus (DM), regardless of confounding factors. European cohort data suggest a lower incidence of DM among individuals with higher habitual flavonoid intake. In the United States, cohort findings hadcorroborate this hypothesis. In a dose-response analysis of a metaanalysis of prospective studies, Liu et al concluded that the intake of $500 \mathrm{mg}$ /day of flavonoids was associated with a $5 \%$ reduction in the risk of DM. ${ }^{18}$

There is a profound transformation in women's intestinal microbiota during pregnancy that can interfere with flavonoid absorption and metabolism. While in vitro and animal models investigations suggest a potential protective effect of flavonoids on Gestational Diabetes Mellitus (GDM), no epidemiological studies have been found to investigate the relationship between the intake of these compounds during pregnancy with excess weight and GDM. These studies resulted in an inverse relationship between the consumption of excess body weight flavonoids and GDM.

\section{Pregnancy induced hypertension}

The growing number of cardiovascular disease patients, including frequently developing hypertension during pregnancy, indicates the need for innovative strategies for more effective prevention and treatment. During childbirth, hypertensive conditions are categorized into 4 groups as per the National High Blood Pressure Education Program Working Group on High Blood Pressure in Childbirth: chronic hypertension, preeclampsia, preeclampsia superimposed on chronic hypertension and gestational hypertension. ${ }^{19-21}$ This definition is more precise and is therefore compared to the older but more widely used Pregnancy-Induced hypertension (PIH).

Because of the reduction of antioxidant capacity, pregnancyinduced hypertension is a state of extremely increased oxidative stress. Oxidative stress is a recognized key factor in the pathogenesis of many diseases, including obstetric complications, as an imbalance between free radical production and antioxidant protection. Impairment of placental blood flow, intrauterine fetal hypoxia, and disruption of oxygen transfer are one of the effects of increased oxidative stress. PIH is a multisystem disorder that involves not only altered oxidant-antioxidant homeostasis, but also inflammatory process and endothelial dysfunction. In addition, during gestation, women are more likely to experience increased oxidative stress, which can lead to adverse neonatal outcomes. ${ }^{22}$

It is suspected that PIH etiology is associated with a systemic inflammatory response, but there are currently no inflammatory markers available to predict this disease. Due to unbalanced regulation of innate and adaptive immune responses, it is suggested that women show an elevated inflammatory response during pregnancy. It is also suspected that PIH etiology may be associated with dysregulation of endogenous defensive pathways. Inflammation is an active process that is controlled by different mediators which control key cellular events to restore homeostasis of the tissue.
Impaired inflammation resolution is likely to play a vital role in the treatment of chronic inflammatory disorders, and one of these is known to be PIH. Since pregnancy-induced hypertension, there have been developments in the understanding of molecular mechanisms responsible for cellular and tissue-specific processes. In addition to the aforementioned factors, certain specific mediators have been identified that play a significant role; PIH is associated with impaired placenta perfusion, resulting in altered angiogenesis marker secretion.

The main aim of treatment of hypertension in pregnant women is to prevent complications throughout pregnancy, during childbirth and postpartum. Much attention is paid to the use of antihypertensive drugs from across different pharmacological groups. ${ }^{23-25}$ Yet, from pharmacokinetic and pharmacodynamic point of view, we should be aware of special conditions for drugs use during pregnancy, namely, the presence of an additional compartment, i.e., the fetalplacental unit.

Drug treatment choices in PIH are limited due to the teratogenic effects of some antihypertensive drugs, such as ACE inhibitors and angiotensin II receptor (AT1) antagonists (sartans). Methyldopa is part of PIH first-line therapy. It is well absorbed and taken per os. This crosses the placenta and hits concentrations of the fetal serum close to those in the mother. Upon intravenous administration, the onset of action is delayed by 1-2 hours and 4-6 hours upon oral administration; it is successful for 6-12 hours.

In the treatment of PIH, many other classes are used, including beta blockers such as metoprolol or labetalol, dihydropyridine derivatives such as nitrendipine or nifedipine, and other vasodilators such as hydralazine and dihydralazine. Nevertheless, methyldopa remains a major component of PIH care due to the documented stability of uteroplacental perfusion and fetalhemodynamics.

\section{Anti-inflammatory effects}

A constrictive effect on the fetal duct is considered to have nonsteroidal anti-inflammatory drugs (NSAIDs). There has been a growing number of cases of fetal ductal constriction not associated with the use of NSAIDs during pregnancy. Previously, we reported that fetal ductal constriction was associated with maternal ingestion of polyphenolrich beverages during pregnancy and that the discontinuation of these substances in most cases resulted in echocardiographic improvement. A variety of foods and drinks, such as herbal teas, grape and orange derivatives, dark chocolate, fruit, and many others, are rich in flavonoids and have been eaten freely throughout gestation period. ${ }^{26-30}$

Considering that constriction of ductus arteriosus in utero may be clinically important in terms of fetal hemodynamic compromise and the potential for neonatal pulmonary hypertension and that its known pathogenic mechanism is inhibition of maternal circulating prostaglandin synthesis in late pregnancy, we hypothesized that polyphenols or flavonoids commonly consumed in food and beverages Therefore, in third-trimester fetuses exposed and not exposed to polyphenol-rich foods (PRFs) and beverages via maternal intake, in a study ductal flow activity and correct ventricular volume were compared.

\section{Utilisation of herbal extract during pregnancy}

One of the approaches used in complementary and alternative medicine is the application of herbal products. Recently, several international studies have been carried out to establish the prevalence of herbal medicine in pregnancy. Such studies enable us to deduce that a growing number of women put their own and their unborn children's health at risk due to lack of knowledge of phytochemical 
properties or effective use of herbal medicinal products, as well as lack of communication with healthcare prices. ${ }^{31}$ There has been a steady increase in interest in the use of herbal products, but very often conflicting or contradictory are the guidelines available.

The prevalence of use of herbal medicine in pregnancy ranges from $7 \%$ to $55 \%$ across different geographic, social and cultural settings and is distinctive across ethnic groups. Very often, herbal products are chosen over prescription medicines because people assume that herbs are healthier for the fetus than modern medicines. Manufacturing processes of herbal medicinal products are very complex because they include non-standardized processes such as plant production, sourcing vegetable raw material from different parts of the world, processing extracts and producing a product in compliance with local standards of good manufacturing practice. ${ }^{32}$

Many studies of epidemiology and intervention strongly suggested that consumption of flavonoids had beneficial effects on vascular health. In various models of vascular disorders, several flavonoids have been investigated showing their antioxidant properties and vasodilator activity, as well as anti-inflammatory, antiatherogenic and antithrombotic effects. Nevertheless, it remains difficult to evaluate the vascular role of dietary flavonoids due to lack of information about their bioavailability, biotransformation, or bioaccumulation, especially during pregnancy. In addition, to measure the antihypertensive effect of dietary flavonoids, biodynamics in the vascular system during pregnancy must be taken into account.

\section{Flavonoids-an essential natural resource}

Plant flavonoids and their conjugates belong to a large family of natural polyphenolic phenylpropanoid-derived compounds commonly found in many varieties of medicinal plants from different families, such as Asteraceae, Lamiaceae, Rutaceae and Passifloraceae, with an estimated 10,000 species. $^{33-35}$ These are structurally composed of two main groups: 2-phenylchromans (flavonoids, including flavanones, flavonols, flavan-3-ols, and anthocyanidins) and3-phenylchromans (isoflavonoids, including isoflavones, isoflavans, and pterocarpans). To civilization, flavonoids were extremely important. These were strongly associated with the health benefits of drinks such as tea and wine.

Flavonoids have a wide spectrum of biological activity and have therefore been commonly used for their anti-inflammatory, ${ }^{36}$ antilipidemic, ${ }^{37}$ antihyperglycemic, ${ }^{38}$ antiviral, ${ }^{39}$ hepatoprotective, ${ }^{40}$ gastric antiulcer, cardioprotective, neuroprotective, antioxidant, and anticancer effects. ${ }^{41}$ Several experiments have shown a wide range of biochemical activities of flavonoids, however their ability to act as antioxidants is the best-described resource. It is well known that the antioxidant activity of flavonoids depends on the atomic structure arrangement of functional groups.

It also synthesizes thromboregulatory molecules and growth factors and reacts to physical and chemical signals. Endothelium also affects the hantatic equilibrium between anticoagulation and thrombosis. Other functions include angiogenesis regulation, wound healing, smooth proliferation of muscle cells, fibrosis, and inflammation. Thus, endothelial dysfunction tends to have a complex relationship with hypertension preceding the development of pathological disorders in the cardiovascular system, while endothelial function improvements can lead to lower blood pressure.

The inverse association between dietary flavonoid intake and chronic degenerative diseases such as cardiovascular diseases (CVD) has been shown not only by various pharmacological studies but also by clinical and epidemiological study. Systematic reviews and metaanalysis of prospective cohort studies have shown that a high intake of flavonoids is associated with lower CVD mortality.

\section{Bioavailability of flavonoids}

Bioavailability can be characterized as "the rate and degree to which a drug product absorbs an active moiety or active ingredient and becomes available at the site of action." This same concept can be extended to flavonoids as the rate of absorption and presence at the site of action is essential for the biological system to be successful. ${ }^{42-46}$ Bioavailability of flavonoids is poor and varies between various subclasses of flavonoids and individual compounds of flavonoids. For example, onion quercetin absorption is four times greater than apples or tea absorption. Flavonoids have poorer bioavailability with complex structures and higher molecular weights. In a clinical study, quercetin from the ingestion of onion powder demonstrated quicker absorption, increased plasma levels and improved bioavailability compared to apple peel powder.

\section{Flavonoids as antioxidants}

Both animal and in vitro experiments, flavonoids showed both antioxidant and prooxidant effects. Because of their ability to contribute hydrogen atom due to their high reactivity of the hydroxyl group, flavonoids are able to scavenge free radicals, resulting in less reactive and stable free radicals. Several flavonoids were found to scavenge superoxide directly while others were found to scavenge peroxynitrite, a radical derived highly reactive oxygen. In vitro flavonoid studies have shown that their antioxidant properties depend on the structural arrangement of functional groups as well as the total number and configuration of hydroxyl groups. ${ }^{47-50}$

The most important determinant of flavonoid ROS scavenging is the $\mathrm{B}$ ring hydroxyl group configuration. Inserting the hydroxy group in ring $\mathrm{A}$ and $\mathrm{C}$, however, has little or no effects on the scavenging effect of superoxide. Certain structural properties of flavonoids in the battle against free radicals include the following: the presence of catechol (orthodihydroxy) in the B ring responsible for electron delocalization, the presence of hydroxy group at positives 5 (A ring) and 3 (C ring) responsible for providing the oxo group with hydrogen bond and the presence of 2,3-double bond in conjunction with a 4-ring.

Indirectly, flavonoids can scavenge free radicals by interacting with specific antioxidant enzymes such as the phosphate system of glutathione and nicotinamide adenine dinucleotide. This can be accomplished through their ability to induce detoxifying enzymes such as $\mathrm{NAD}(\mathrm{P}) \mathrm{Hquinone}$ oxidoreductase, and S-transferase glutathione, which are the key enzymes of protection against oxidative stress and electrophilic toxicants. Hydroxy-functional group flavonoids at positionC-3 (e.g. quercetin) are more efficient in triggering certain antioxidant mechanisms and inducing the electrophile-responsive feature.

Flavonoids have demonstrated the ability to chelate iron and copper, thus eliminating a causal factor in free radical formation. A number of oxidizing agents (such as acrolein) that affected the release of iron in its redox state in the erythrocyte membrane found quercetin to prevent oxidative cellular injury. The binding site for trace metal in the flavonoid structure, according to a review, is the group of 3-hydroxyl and 4-oxo in the $\mathrm{C}$ ring, catechol moiety in the $\mathrm{B}$ ring, and 4-oxo and5-hydroxyl groups in the $\mathrm{A}$ and $\mathrm{C}$ ring. Because of its ability to chelate cupric cation, the main contributor of the metal chelation is the catechol moiety in the $\mathrm{B}$ ring. 
Flavonoids have the ability to block superoxide-generating enzymes such as xanthine oxidases and protein kinase C. Also, flavonoids have been shown to be effective cyclooxygenase, microsomal succin-oxidase, lipoxygenase, and NADH oxidase inhibitors. Based on the structural structure of flavonoids, luteolin was found to be the most potent inhibitor of xanthine oxidase, a member of the subclass flavone, which eventually led to a reduction in oxidative cell injury.

Certain antioxidant properties of flavonoids include: increased uric acid levels, mitigation of nitric oxide oxidative stress, reduction of $\alpha$-tocopheryl radicals, and alteration of low molecular antioxidant prooxidant properties. Flavonoids in edible plants and fruits are also known to have protective effects against oxidative stress primarily by preventing intracellular ROS formation. ${ }^{51-53}$ It is interesting to note that scientific research now focuses on the use of natural food supplements to avoid many of these disorders. However, several studies on the antioxidant effects of flavonoids in cancer, cardiovascular diseases, and neurodegeneration have been conducted.

\section{Role of flavonoids in vasculo-endothelial function}

Endothelial dysfunction is a major event in pre-eclampsia, cardiovascular disease, and cancer pathogenesis. Healthy endothelial cells facilitate vascular repairs and increase the invasion of trophoblasts by maintaining vascular homeostasis and vascular tone control. Through managing the output of vasodilators such as nitric oxide, this maintenance is done. Dietary flavonoids increase the level of nitric oxide that enhances endothelial function by increasing endogenous nitric oxide production and decreasing its breakdown. Human clinical trials have shown that in acute and short-term action, flavonoids improve endothelial functions. ${ }^{54-57}$ Improving endothelial dependent and nitric oxide dependent vasodilation has been suggested as one of the possible mechanisms for involving flavonoids in endothelial and vascular function. The endothelial function change may also lead to lower blood pressure.

\section{Role of flavonoids in glucose and lipid metabolism}

In order to maintain various physiological, homeostatic and cellular processes in the human body, lipids and glucose are essential macromolecules required. In pathogenesis of several diseases including obesity, cardiovascular diseases, diabetes and inflammation, dysfunction in lipid, lipoprotein and glucose metabolism has been associated. It has been found that citrus flavonoids (especially naringin) exhibit lipid-lowering properties in humans by significantly reducing levels of plasma triglyceride and apolipoprotein $\mathrm{B}$ in hypertriglyceridemic patients.

In a clinical trial involving hypercholesterolemic patients, citrus flavonoid (naringin) was found to significantly decrease plasma low density lipoprotein (LDL). For human subjects, the lipid reduction mechanism of citrus flavonoids has been fully established. Other research, however, had an inhibitory effect on the reductase and acyl CoA of hepatic HMG-CoA (3-hydroxy-3methyl-glutarylcoenzymeA).Cholesterol lowering effects of flavonoids were probably due to their direct binding to or due to interaction with receptors of oestrogen. ${ }^{58-60}$

\section{Flavonoids as anti-cholinesterase activity}

Acetylcholinesterase (AChE) is a crucial enzyme in the central nervous system and its inhibition leads to increased rates of neural acetylcholine, one of the treatments for mild to moderate $\mathrm{AD}$ symptomatic relief. Therefore, cholinesterase inhibition is one of the central focus of drug development in the fight against AD. For their anticholinesterase activity, a number of flavonoids have been identified. Studies conducted on various flavonoids such as quercetin, rutin, kaempferol 3-O- $\beta$-D-galactoside and macluraxanthone in vitro have shown that quercetin and macluraxanthone have a concentrationdependent ability to inhibit $\mathrm{AChE}$ and butyrylcholinsterase (BChE). Macluraxanthone was found to be the most active and common inhibitor of both enzymes with an inhibitory concentration of 50 percent (IC50) of 8.47 and $29.8 \mu \mathrm{M}$ respectively. The kinetic enzyme studies showed that quercetin competitively inhibited both enzymes, while macluraxanthone was non-competitiveagainstAChE and competitive against $\mathrm{BChE}$. Molecular docking tests of these two compounds were performed at active sites of both enzymes to gain insight into the intermolecular interactions. The docking studies have shown that macluraxanthone binds to both enzymes much more strongly than quercetin. The docking experiments have shown that macluraxanthone binds to both enzymes much more strongly than quercetin. ${ }^{61-63}$

\section{Mechanism of flavonoids}

Nearly any group of flavonoids will act as antioxidants. Flavones and catechins have been reported to be the most effective flavonoids to protect the body from reactive oxygen species. The damage caused by free radicals and reactive oxygen species, which are created during normal oxygen metabolism or triggered by exogenous damage, continues to threaten body cells and tissues. The mechanisms and sequence of events that interfere with cellular functions by free radicals are not fully understood, but one of the most important events appears to be lipid peroxidation, resulting in damage to the cell membrane. ${ }^{64-66}$

Such cell damage causes a change in the cell's net charge, increasing the osmotic pressure, causing swelling and eventually death of the cell. Free radicals may attract different inflammatory mediators, leading to a general inflammatory response and damage to the tissue. Living organisms have developed a number of successful mechanisms to defend themselves from reactive oxygen species. The body's antioxidant defense mechanisms include enzymes such as superoxide dismutase, catalase, and glutathione peroxidase, as well as non-enzymic equivalents such as glutathione, ascorbic acid, and $\alpha$-tocopherol. ${ }^{67}$

The increased production during injury of reactive oxygen species results in endogenous scavenging compounds being consumed and depleted. The endogenous scavenging compounds may have an additive effect on flavonoids. The theoretical order of affinity between flavonoids and amino acid residues tends to have significant implications in the theoretical predictions of flavonoid-protein interactions as a high-quality approach to understanding flavonoids ' biological activity.

\section{Radical scavenging}

In various ways, flavonoids can avoid injury caused by free radicals, and one way is to scavenge free radicals directly. Radical oxidization of flavonoids results in a more stable, less reactive radical. In other words, by reacting with the radical's reactive element, flavonoids stabilize the reactive oxygen species. As explained in the following equation as given by Korkina \& Afanasev, radicals are made inactive due to the high reactivity of the hydroxyl group of flavonoids. ${ }^{68-70}$ 


\section{Flavonoid $(\mathrm{OH})+r(\mathrm{O})+\mathbf{R H}$}

\section{Flavonoids to treat neurodegenerative disorders}

Recent studies on various plant metabolites have shown that flavonoids can play a key role in the brain's enzyme and receptor systems, with significant effects on the central nervous system, such as neurodegeneration protection associated with $\mathrm{AD}$ and Parkinson's disease. ${ }^{71-74}$ Flavonoids can inhibit enzymes as there are several reports of inhibitory enzymes in preventive neurodegenerative diseases such as aldose reductase, $\mathrm{XO}$, phosphodiesterase, $\mathrm{Ca} 2$ + ATPase, lipoxygenase, and COX. A significant amount of work has been done to look for appropriate and experimental flavonoids for medicinal use in $\mathrm{AD}$ using the molecular docking technique.

Most of them had more potent anti-AChE inhibitory activities than rivastigmine, an $\mathrm{AD}$ drug. It was also reported that the isoflavone skeleton would be a promising structural basis for the development of novel inhibitors of AChE. A number of flavonoids were tested using molecular docking studies to lower Alzheimer's production of $A \beta$. It has been stated that a strong correlation exists between flavonoids and mechanism-related inhibitions of NF- $\pi \mathrm{B}$. When working on the molecular docking of flavones as BACE-1 inhibitors, flavonoids have been found to inhibit BACE-1 activity by flavonoid interactions with the catalytic center BACE- $1 .^{75}$

\section{Applications and functions of flavonoids}

Plants produce a wide variety of organic compounds, the vast majority of which do not appear to be directly involved in growth and development. Traditionally referred to as secondary metabolites (flavonoids), these substances are often distributed differently within the plant kingdom among small taxonomic groups. The flavonoids are known as alkaloids, terpenoids and phenolics in different classes. In the human body, flavonoids perform a variety of defensive roles. Most flavonoids have developed as bioactive compounds that interact with nucleic acid or proteins and have pharmacological and antimicrobial or insecticidal properties. ${ }^{76-80}$

Therefore, flavonoids are of use in medicine as therapeutics and in agriculture as pesticides at the same time. In vitro technology has provided new insight into the ability of plant cell tissue culture to generate the same useful chemical compounds as the parent plant's. The development of methods for flavonoid processing in plant tissue culture has flourished beyond expectations. Plant tissue cultivation is an aseptic technique whereby proper management of nutrients, crop conditions and supply of phyto-hormone which allow the required quality and quantity of plants and metabolites to be produced. ${ }^{81}$

It is possible to obtain output of the desired compounds at levels comparable to that of the plant with the culture of differentiated cells. ${ }^{82}$ Flavonoids are related to a wide range of health-promoting effects. In a number of nutraceutical, biomedical, medical and cosmetic uses, they are an indispensable part. This is due to their antioxidant, anti-inflammatory, anti-mutagenic and anti-carcinogenic properties combined with their ability to modulate the key function of cellular enzymes. ${ }^{83}$

In plants, flavonoids act as antioxidants, antimicrobials, photoreceptors, visual attractors, repellents for feeding and light screening. Several studies have suggested that there are biological functions of flavonoids, including anti-allergenic, antiviral, antiinflammatory and vasodilating acts. Much interest, however, has been devoted to flavonoids' antioxidant activity due to their ability to suppress free radical formation and scavenge free radicals. ${ }^{84}$
Over recent years, the ability of flavonoids to act as antioxidants over vitro has been the subject of several studies and important structure-activity relationships have been identified with antioxidant activity. ${ }^{85}$

A number of studies have been performed on antioxidant properties in relation to various flavonoids, and these studies have stressed that flavonoids can be used as potential drugs to prevent oxidative stress. Antioxidants are compounds that protect cells against the reactive oxygen species' oxidative effect and result in oxidative stress due to the compromised balance between these reactive oxygen species and antioxidants. Oxidative stress can result in cellular damage that is associated with various health conditions such as diabetes, cancer, CVD, neurodegenerative disorders, and ageing. ${ }^{86}$

Antioxidants interact with radical-producing processes and increase endogenous antioxidant activity, preventing these free radicals from damage to cells. Flavonoids have been shown to be highly effective in preventing lipid peroxidation, and lipid peroxidation, along with aging, is responsible for various diseases such as atherosclerosis, diabetes, hepatotoxicity and inflammation. Research has shown that quercetin helps inhibit the peroxidation of lipids. Besides quercetin, there are other flavonoids including myricetin, quercetrin and rutin that help inhibit superoxide radical's growth. ${ }^{87}$

Flavonoids have also been known for their antimicrobial activity, and many researchers have isolated and identified flavonoid structures that have antimicrobial, antiviral and antibacterial properties. Most flavonoids are now used widely in the fields of nutrition, food safety and health because of this product. Flavonoids including quercetin, naringin, hesperetin, and catechin have variable antiviral activity. ${ }^{88-90}$ We impact the replication and invasion of some viruses of RNA and DNA. Quercetine and apigenin are among the most widely studied flavonoids believed to have antibacterial efficacy.

Many flavonoids are hormone-like and have a resemblance to steroid hormones, particularly with oestrogen. In fruits and vegetables, tea, red wine and cereals, these flavonoids are present. Hormone-like steroids are well known for their protection from various chronic diseases, especially oestrogen, which has neuroprotective effects on the brain. A variety of flavonoids have been tested in clinical trials, such as genistein, daidzein and equol, to determine their oestrogenic effect. The studies have established their ability to treat various chronic diseases such as cancer, cardiovascular disorders and osteoporosis.

\section{Utilisation of nanomedicine in varied applications of flavonoids}

Applying nanotechnologies to cancer therapy may increase the solubility and/or bioavailability of natural or synthetic bioactive compounds and provide other potential benefits in cancer therapy, including selective targeting. Various studies tried to analyze in vivo research on nanoparticles (NPs) anti-cancer activity obtained from flavonoids extracted from food. ${ }^{92}$ A total of 60 studies have been reported from a systematic search. Most experiments included epigallocatechin-3-O-gallate flavanol and quercetin flavonol in both delivery and co-delivery systems (with anti-cancer drugs).

In addition, several studies have studied the effects of other flavonoids, including anthocyanins aglycones anthocyanidins, flavanones, flavones, and isoflavonoids. In both xenografts and chemical-induced animal models of cancerogenesis, NPs inhibited tumor growth. Encapsulation increased bioavailability and/or decreased toxicity for both flavonoidsand/or co-delivered drugs such 
as doxorubicin, docetaxel, paclitaxel, honokiol and vincristine. In addition, in molecular guided nanosystems, flavonoids have been successfully applied. ${ }^{93-95}$

Lifestyle factors, such as changes in dietary intake, are generally known to be closely related to the incidence of cancer. ${ }^{96-98}$ Population and laboratory studies have collected evidence supporting a positive relationship between dietary consumption of fruit and vegetables and reduced risk of different cancers. The positive correlation between flavonoid intake and cancer incidence has been demonstrated in several in vitro and in vivo studies in addition to various clinical trials and meta-analyses. ${ }^{99-102}$

Although the underlying chemopreventive and chemotherapeutic mechanisms are needed to be elucidated, flavonoids have been thoroughly investigated as to how they modulate various key elements in cellular signal transduction pathways and their effect on cell fate determination processes. ${ }^{103-105}$ Most dietary flavonoids not only inhibit the growth of tumor cells but also promote cell differentiation by quenching oxidative stress and inflammatory response.

Cancer therapy has become a multidisciplinary challenge requiring close collaboration between physicians, molecular biologists, scientists of materials and biomedical engineers. ${ }^{106-108}$ Chemotherapy's basic purpose is to improve patients' survival time and quality of life. Conventional chemotherapeutic agents are not clearly administered in the body and due to the severe side effects in the vital organs, the effective dose of chemotherapeutic agents within a solid tumor is not readily available. ${ }^{109}$

Through changing the pharmacokinetic parameters and reducing systemic toxicity, nanotechnologies often offer the possibility of re-entering many drugs on the market, including poorly soluble agents, highly toxic agents and bioactive macromolecules that were previously unable to be used. Indeed, advances in nanomedicine in cancer therapy have rapidly become clinical practice. ${ }^{110,111}$ Some novel formulations based on nanotechnology such as polyethylene glycol (PEG) ylated-liposomal doxorubicin (Doxil), liposomal daunorubicin (DaunoXome), liposomal cytarabine (DepoCyt) and albuminbound particle paclitaxel (Abraxane) reportedly demonstrated the effectiveness of this new class of agents.

Numerous studies illustrated the details of the flavonoids involved in the synthesis of MNPs and their biomedical effectiveness. Recently, a study found that the total flavonoids present in the Alternanthera tenella and Coriandrum sativum leaf extracts were largely involved in AgNPs synthesis; and demonstrated antiacne, antidandruff, and anti-breast cancer activity against Propionibacterium acnes, Malasseziafurfur, and human breast adenocarcinoma cells.

Zuas et al reported that the five water-soluble flavonoids present in Myrmecodiapendan extract were primarily responsible for the $\mathrm{Ag}+$ to AgNP reduction process. Dalbergia spinosa leaf extract flavonoids could be adsorbed to the metal surface by interacting with carbonyl groups or electrons and exhibiting increased anti-inflammatory and antibacterial activities (against Bacillus subtilis, Pseudomonas aeruginosa, Staphylococcus aureus, and Escherichia coli)

Raghunandan et al noticed that AuNPs derived extract of flavonoids functionalized clove buds showed anticancer activity against various cancer cells. Aromal and Philip conducted AuNPs synthesis using the seed extract Trigonella foenumgraecum and concluded that flavonoids are powerful reduction agents for chloroauric acid $\left(\mathrm{HAuCl}_{4}\right)$. The flavonoids in the Ranunculus muricatus aqueous extract interact with metal ions through covalent co-ordinate bonding for $\mathrm{Au} / \mathrm{TiO} 2$ nanocomposite formation, and further inhibit effectively organisms like $S$. aureus and E. coli.

Apigenin is reportedly found to be a bioflavonoid citrus which has antioxidant, anti-inflammatory, and anticancer activity. Kasthuri et al synthesized AuNPs and AgNPs using apiine (apigenin glycoside), which was extracted from Lawsoniainermis and expected to be an effective nano-drug for cancer treatment. Baicalein is the major bioactive flavanoid in the treatment of methicillin-resistant $S$. aureus, which has remarkable synergetic effects with tetracycline and b-lactams. In addition, it has been reported that baicalein attenuates the quorum sensing-controlled virulence factors including the formation of biofilms in P. aeruginosa. Rajkumari et al reported AuNPs mediated on baicalein synthesis and confirmed their enhanced antibiofilm activity against opportunistic pathogen, $P$. aeruginosa in comparison to baicalein.

Dihydromyricetine (DMY), is a flavonol type. It has multiple biological effects including hypoglycemic activity, antioxidant, and antibacterial. Guo et al synthesized AuNPs using DMY without adding any surfactants, capping agents or templates external to them. Moreover, the experimental approach suggests that the AuNPs synthesis mediated by flavonoids is simple, rapid, cost-effective and reproducible. Genistein, an isoflavonoid which occurs naturally, has both anticancer and antiangiogenic properties. Stolarczyk et al recently observed that genistein reduces $\mathrm{Au}^{3+}$ to spherical $\mathrm{Au}^{0}$ nanocrystallite.

\section{Flavonoids and its role in treatment of COVID-I9}

Coronaviruses (CoVs) are an etiologic agent of serious infections in both humans and animals, which can cause disorder not only in the respiratory tract, but also in the digestive tract and in the system. Previous CoV studies have reported that CoVs may infect certain animal species, including mammals, avian species and reptiles. The new CoV strain was identified at the end of 2019, initially named 2019-nCoV, and emerged during an outbreak in Wuhan, China. Coronaviruses (CoVs) are an etiologic agent of serious infections in both humans and animals, which can cause disorder not only in the respiratory tract, but also in the digestive tract and in the system. Previous $\mathrm{CoV}$ studies have reported that $\mathrm{CoVs}$ may infect certain animal species, including mammals, avian species and reptiles. The new CoV strain was identified at the end of 2019, initially named 2019-nCoV, and emerged during an outbreak in Wuhan, China. Liu et al (2020) successfully crystallized the main protease $\left(\mathrm{M}^{\mathrm{pro}}\right)$ of COVID-19, which is a potential drug target.

Other reported antiviral treatments for human pathogenic CoVs include nucleoside analogs, neuraminidase inhibitors, remdesivir, umifenovir (arbidol), tenofovir disoproxil (TDF) and lamivudine (3TC). A separate investigation conducted by Xu et al. (2020) indicated that, of the 4 drugs tested (nelfinavir, pitavastatin, perampanel, and praziquantel), nelfinavir was identified as the best potential inhibitor of COVID-19 Mpro based on the binding of free energy calculations using generalized Born molecular mechanics and surface area solution (MM / GBSA) model and solvent interactions.

At present, COVID-19 has emerged in the human population, in China, and is a potential threat to global health worldwide. However, there is currently no approved drug for the treatment of the disease. The drugs currently available for treatment with COVID-19 primarily act on the main protease $\left(\mathrm{M}^{\text {pro }}\right)$. In a study, they attempted to examine a number of medicinal herbal compounds that could be used to inhibit the pathway of infection with COVID-19. Nelfinavir, lopinavir, 
kaempferol, quercetin, luteolin-7-glucoside, demethoxycurcumin, naringenin, apigenin-7-glucoside, oleuropein, curcumin, catechin and epicatechin-gallate had the lowest binding energy and inhibition constants.

The affinity of kaempferol bonds is higher compared to other compounds. Therefore, this study suggested that nelfinavir and lopinavir may represent potential treatment options, and kaempferol, quercetin, luteolin-7-glucoside, demethoxycurcumin, naringenin, apigenin-7-glucoside, oleuropein, curcumin, catechin and epicatechingallate were the most recommended compounds found in medicinal plants that could act as potential inhibitors of COVID-19 $\mathrm{M}^{\text {pro }}$. However, further research is needed to investigate the potential uses of medicinal plants containing these compounds.

\section{Futuristic role of flavonoids}

Over the past 10 years, flavonoids have been given a lot of coverage in the literature and a number of possible beneficial effects have been elucidated. In vitro and in silico experiments, however, a number of studies were carried out. More studies are therefore needed in order to enhance the utility of flavonoids in the diet for better human health. Due to the heterogeneity of the different molecular structures and the scarcity of bioavailability data, flavonoid analysis is complex. In addition, there are inadequate methods available to assess in vivo oxidative damage and it is still difficult to measure objective endpoints. ${ }^{91}$ Better analytical techniques are needed to enable more data to be collected on absorption and excretion. In particular, data on the long-term effects of chronic consumption of flavonoids are sparse. Numerous reports also highlighted the need for molecular docking studies to identify potential flavonoid molecules for their use in the treatment of various health system disorders. An important area of future research is the interaction of flavonoids with receptor molecules during the treatment of acute and chronic diseases.

\section{Conclusion}

Flavonoids are considered to be one of the most vital compounds which had proved to show anti-diabetic, anti-cancerous, anti-oxidant, anti-inflammatory and also in treatment of Neurodegenerative disorders. There are various applications like nanoparticle mediated delivery of flavonoids to decimate tumor cells. There are also studies which proved that flavonoids aid in treating pregnancy induced hypertension. Recently, there are also reports which proved that flavonoids show anti-cholinesterase activity. Flavonoids still shows great potential in further research which would prove beneficial in curing heinous diseases in future.

\section{Acknowledgments}

The authors are thankful to GITAM (Deemed to be University), Visakhapatnam, Andhra Pradesh for making available facilities to carry out this work.

\section{Conflicts of interest}

The authors declare no conflicts of interest.

\section{References}

1. Townsend R, O'Brien P, Khalil A. Current best practice in the management of hypertensive disorders in pregnancy. Integrated Blood Pressure Control. 2016;9:79-94.

2. Mammaro A, Carrara S, Cavaliere A, et al. Hypertensive Disorders of Pregnancy. Journal of Prenatal Medicine. 2009;3(1):1-5.
3. Luanni VB, Ota E, Togoobaatar G, et al. Risk factors of pre-eclampsia/ eclampsia and its adverse outcomes in low- and middleIncome countries: a WHO secondary analysis. PLoS One. 2014;9(3):e91198.

4. Fauci AS, Braunwald E, Kasper DL. Disorders of intermediary metabolism. In Harrison's Principles of Internal Medicine, Fauci AS, Braunwald E, and Kasper DL, Eds. McGraw-Hill: New York, NY, USA; 2008. 2426-2429 p.

5. Masoura S, Kalogiannidis IA, Gitas G, et al. Biomarkers in pre-eclampsia: A novel approach to early detection of the disease. Journal of Obstetrics \& Gynaecology. 2012;32(7):609-616.

6. Myatt L, Roberts JM. Preeclampsia: Syndrome or Disease? Current Hypertension Reports. 2015;17(11):83.

7. Rehfeldt ME, Eklund J, Struck et al. Relaxin-2 connecting peptide (proRLX2) levels in second trimester serum samples to predict preeclampsia. Pregnancy Hypertension: An International Journal of Women's Cardiovascular Health. 2018;11:124-128.

8. Schaefer C, Peters P, Miller RK. Drugs during Pregnancy and Lactation. Treatment Options and Risk Assessment. Elsevier: Oxford, UK; 2007.

9. Myatt L, Webster RP. Is vascular biology in preeclampsia better? Journal of Trombosis and Haemostasis. 2007;7(3):375-384.

10. Middeldorp S. Pregnancy Failure and Heritable Trombophilia. Seminars in Hematology. 2007;44(2):93-97.

11. Baumwell S, Karumanchi SA. Pre-eclampsia: clinical manifestations and molecular mechanisms. Nephron Clinical Practice. 2007;106(2):c72-c81.

12. Fatini C, Sticchi E, Gensini F, et al. Endothelial nitric oxide synthase gene infuences the risk of pre-eclampsia, the recurrence of negative pregnancy events, and the maternal-fetalfow. Journal of Hypertension. 2006;24(9):1823-1829.

13. Napolitano M, Miceli F, Calce A, et al. Expression and relationship between endothelin-1 messenger ribonucleic acid (mRNA) and inducible/ endothelial nitric oxide synthase mRNA isoforms from normal and preeclamptic placentas. The Journal of Clinical Endocrinology \& Metabolism. 2000;85(6):2318-2323.

14. Powe CE, Levine RJ, Karumanchi SA. Preeclampsia, a disease of the maternal endothelium: the role of antiangiogenic factors and implications for later cardiovascular disease. Circulation. 2011;123(24):2856-2869.

15. Tomimatsu T, Mimura K, Endo M, et al. Pathophysiology of preeclampsia: An angiogenic imbalance and long-lasting systemic vascular dysfunction. Hypertension Research. 2017;40(4):305-310.

16. Słowinski T, Neumayer H, Stolze T, et al. Endothelin system in normal and hypertensive pregnancy. Clinical Science. 2002;103(s48):446S-449S.

17. Baksu B, Davas I, Baksu A, et al. Plasma nitric oxide, endothelin-1 and urinary nitric oxide and cyclic guanosine monophosphate levels in hypertensive pregnant women. International Journal of Gynecology and Obstetrics. 2005;90(2):112-117.

18. Amaral LM, Cunningham MW, Cornelius DC, et al. Preeclampsia: long-term consequences for vascular health. Vascular Health and Risk Management. 2015;11:403-415.

19. Possomato-Vieira JS, Khalil RA. Mechanisms of Endothelial Dysfunction in Hypertensive Pregnancy and Preeclampsia. Advances in Pharmacology. 2016;77:361-431.

20. Bakrania B, Duncan J, Warrington JP, et al. The endothelin type a receptor as a potential therapeutic target in preeclampsia. International Journal of Molecular Sciences. 2017;18(3):1-8.

21. Granger JP, Spradley FT, Bakrania BA. The Endothelin System: A Critical Player in the Pathophysiology of Preeclampsia. Current Hypertension Reports. 2018;20(4):32. 
22. Calabro V, Litterio MC, Fraga CG, et al. Efects of quercetin on heart nitric oxide metabolism in 1 -NAME treated rats. Archives of Biochemistry and Biophysics. 2018;647:47-53.

23. Pereira SC, Parente JM, Belo VA, et al. Quercetin decreases the activity of matrix metalloproteinase-2 and ameliorates vascular remodeling in renovascular hypertension. Atherosclerosis. 2018;270:146-153.

24. Ajibade TO, Oyagbemi AA, Omobowale TO, et al. Quercetin and Vitamin C Mitigate Cobalt Chloride-Induced Hypertension through Reduction in Oxidative Stress and Nuclear Factor Kappa Beta (NF-Kb) Expression in Experimental Rat Model. Biological Trace Element Research. 2017; 175(2):347-359.

25. Plotnikov MB, Aliev OI, Sidekhmenova AV, et al. Modes of Hypotensive Action of Dihydroquercetin in Arterial Hypertension. Bulletin of Experimental Biology and Medicine. 2017;162(3):353-356.

26. Morales-Cano D, Menendez C, Moreno E, et al. The flavonoid quercetin reverses pulmonary hypertension in rats. PLoS One. 2014;9(12):e114492.

27. Galindo P, Gonzalez-Manzano S, Zarzuelo MJ, et al. Diferent cardiovascular protective efects of quercetin administered orally or intraperitoneally in spontaneously hypertensive rats. Food \& Function. 2012;3(6):643-650.

28. Gao H, Chen C, Huang S, et al. Quercetin attenuates the progression of monocrotaline-induced pulmonary hypertension in rats. Journal of Biomedical Research. 2012;26(2):98-102.

29. Monteiro MMO, Franca-Silva MS, Alves NFB, et al. Quercetin improves barorefex sensitivity in spontaneously hypertensive rats. Molecules. 2012;17(11):12997-13008.

30. Romero M, Jimenez R, Hurtado B, et al. Lack of benefcial metabolic efects of quercetin in adult spontaneously hypertensive rats. European Journal of Pharmacology. 2010;627(1-3):242-250.

31. Carlstrom J, Symons JD, Tzu CW, et al. A quercetin supplemented diet does not prevent cardiovascular complications in spontaneously hypertensive rats. Journal of Nutrition. 2007;137(3):628-633.

32. Duarte J, Perez-Palencia R, Vargas F, et al. Antihypertensive efects of the favonoid quercetin in spontaneously hypertensive rats. British Journal of Pharmacology. 2001;133(1):117-124.

33. Plotnikov MB, Aliev OI, Anishchenko AM, et al. Parameters of cerebral cortex capillary network in SHR rats during the development of arterial hypertension and stable high blood pressure. Rossiiskii Fiziologicheskii Zhurnal. 2016;102(5):558-566.

34. Slashcheva GA, Rykov VA, Lobanov AV, et al. Dihydroquercetin Does Not Afect Age-Dependent Increase in Blood Pressure and AngiotensinConverting Enzyme Activity in the Aorta of Hypertensive Rats. Bulletin of Experimental Biology and Medicine. 2016;161(5):670-673.

35. Brull V, Burak C, Stofel-Wagner B, et al. Acute intake of quercetin from onion skin extract does not infuence postprandial blood pressure and endothelial function in overweightto-obese adults with hypertension: a randomized, doubleblind, placebo-controlled, crossover trial. European Journal of Nutrition. 2017;56(3):1347-1357.

36. Biesinger S, Michaels HA, Quadros AS, et al. A combination of isolated phytochemicals and botanical extracts lowers diastolic blood pressure in a randomized controlled trial of hypertensive subjects. European Journal of Clinical Nutrition. 2016;70(1):10-16.

37. Dower JI, Geleijnse JM, Gijsbers L, et al. Efects of the pure favonoids epicatechin and quercetin on vascular function and cardiometabolic health: a randomized, double-blind, placebo-controlled, crossover trial. American Journal of Clinical Nutrition. 2015;101(5):914-921.
38. Dower JI, Geleijnse JM, Gijsbers L, et al. Supplementation of the pure favonoids epicatechin and quercetin afects some biomarkers of endothelial dysfunction and infammation in (pre)hypertensive adults: a randomized double-blind, placebocontrolled, crossover trial. Journal of Nutrition. 2015;145(7):1459-1463.

39. Brull V, Burak C, Stofel-Wagner B, et al. Effects of a quercetin-rich onion skin extract on $24 \mathrm{~h}$ ambulatory blood pressure and endothelial function in overweight-to-obese patients with (pre-)hypertension: a randomised double-blinded placebo-controlled cross-over trial. British Journal of Nutrition. 2015;114(8):1263-1277.

40. Perez A, Gonzalez-Manzano S, Jimenez R, et al. Tefavonoid quercetin induces acute vasodilator efects in healthy volunteers: Correlation with beta-glucuronidase activity. Pharmacological Research. 2014;89:11-18.

41. Larson A, Witman MAH, Guo Y, et al. Acute, quercetininduced reductions in blood pressure in hypertensive individuals are not secondary to lower plasma angiotensinconverting enzyme activity or endothelin-1: Nitric oxide. Nutrition Research. 2012;32(8):557-564.

42. Serban MC, Sahebkar A, Zanchetti A, et al. Efects of quercetin on blood pressure: a systematic review and meta-analysis of randomized controlled trials. Journal of the American Heart Association. 2016;5(7):10-1161.

43. Draganovic D, Lucic N, Jojic D, etal. Correlation of oxidative stress markers with ultrasound and cardiotocography parameters with hypertension induced pregnancy. Acta Informatica Medica. 2017;25(1):19-23.

44. Bharadwaj S, Bhat VB, Vickneswaran V, et al. Oxidative stress in preeclamptic mother-newborn dyads and its correlation with early neonatal outcome-a case control study. The Journal of Maternal-Fetal and Neonatal Medicine. 2018;31(12):1548-1553.

45. Cornelius DC. Preeclampsia: From infammation to immunoregulation. Clinical Medicine Insights: Blood Disorders. 2018;11:1-6.

46. Liberis A, Stanulov G, Chafouz Ali E, et al. Pre-eclampsia and the vascular endothelial growth factor: A new aspect. Clinical and Experimental Obstetrics \&Gynecology. 2016;43(1):9-13.

47. Chau K, Hennessy A, Makris A. Placental growth factor and preeclampsia. Journal of Human Hypertension. 2017;31(12):782-786.

48. Hentschke MR, Ruszkowski RA, da Costa BE, et al. Placental growth factor and severity of preeclampsia: biomarkers, prediction of preeclampsia. Pregnancy Hypertension: An International Journal of Women's Cardiovascular Health. 2016;6(3):156.

49. Manriquez Rocha B, Mbofana F, Loquiha O, et al. Early diagnosis of preeclampsia using placental growth factor: An operational pilot study in Maputo, Mozambique. Pregnancy Hypertension: An International Journal of Women's Cardiovascular Health. 2018;11:26-31.

50. Xu B, Nakhla S, Makris A, et al. TNF- $\alpha$ inhibits trophoblast integration into endothelial cellular networks. Placenta. 2011;32(3):241-246.

51. Smith S, Francis R, Guilbert L, et al. Growth factor rescue of cytokine mediated trophoblast apoptosis. Placenta. 2002;23(4): 322-330.

52. Marwa BAG, Raguema N, Zitouni H, et al. FGF1 and FGF2 mutations in preeclampsia and related features. Placenta. 2016;43: 81-85.

53. Hohlagschwandtner M, Knofer M, Ploner M, et al. Basic fbroblast growth factor and hypertensive disorders in pregnancy. Hypertension in Pregnancy. 2002;21(3):235-241.

54. Frampton GK, Jones J, Rose M, et al. Placental growth factor (Alone or in combination with soluble fmslike tyrosine kinase 1) as an aid to the assessment of women with suspected pre-eclampsia: Systematic review and economic analysis. Health Technology Assessment. 2016;20(87):1192. 
55. Duckworth S, Grifn M, Seed PT, et al. Diagnostic Biomarkers in Women with Suspected Preeclampsia in a Prospective Multicenter Study. Obstetrics \& Gynecology. 2016;128(2):245-252.

56. Kaluba-Skotarczak A, Magiełda J, Romała A, et al. Importance of polymorphic variants of Tumour Necrosis Factor-gene in the etiology of Intrauterine Growth Restriction. Ginekologia Polska. 2018;89(3):160168.

57. Lowe SA, Rubin PC. The pharmacological management of hypertension in pregnancy. Journal of Hypertension. 1992;10(3):201-207.

58. Seremak-Mrozikiewicz A, Drews K. Methyldopa in therapy of hypertension in pregnant women. Ginekologia Polska. 2004;75(2):160 165.

59. Picaud A, Walter P, de Preville G, et al. Fatal toxic hepatitis in pregnancy. A discuss the role of methyldopa. Journal de Gynecologie Obstetrique et Biologie de la Reproduction. 1990;19(2):192-161.

60. Sibai BM. Treatment of hypertension in pregnant women. The New England Journal of Medicine. 1996;335(4):257-265.

61. Caton AR, Bell EM, Druschel CM, et al. Antihypertensive medication use during pregnancy and the risk of cardiovascular malformations. Hypertension. 2009;54(1):63-70.

62. Xie RH, Guo Y, Krewski D, et al. Trends in using betablockers and methyldopa for hypertensive disorders during pregnancy in a Canadian population. European Journal of Obstetrics \&Gynecology and Reproductive Biology. 2013;171(2):281-285.

63. Adrover R. Hepatotoxicity from Alpha-Methyldopa During Pregnancy: Two Case Reports. Journal of Clinical Gastroenterology and Treatment. 2016;2(3).

64. Aldomet (methyldopa) side effects; 2018.

65. Ghanem FA, Movahed A. Use of antihypertensive drugs during pregnancy and lactation. Cardiovascular Therapeutics. 2008;26(1):38-49.

66. Singh P, Mishra A, Goswami S, et al. Hypertension and herbal plant for its treatment: a review. Indian Journal of Research in Pharmacy and Biotechnology. 2015;3(5):358-366.

67. Tome-Carneiro J, Visioli F. Polyphenol-based nutraceuticals for the prevention and treatment of cardiovascular disease: review of human evidence. Phytomedicine. 2016;23(11):1145-1174.

68. Schurger N, Klein E, Hapfelmeier A, et al. Demand for integrative medicine among women in pregnancy and childbed: a German survey on patients' needs. BMC Complementary and Alternative Medicine. 2018;18(1):187.

69. Kennedy DA, Lupattelli A, Koren G, et al. Herbal medicine use in pregnancy: results of a multinational study. BMC Complementary and Alternative Medicine. 2013;13(1):1-10

70. John LJ, Shantakumari N. Herbal medicines use during pregnancy: A review from the middle east. Oman Medical Journal. 2015;30(4):229236.

71. Akour A, Kasabri V, Aff FU, et al. The use of medicinal herbs in gynecological and pregnancy-related disorders by Jordanian women: a review of folkloric practice vs. evidence-based pharmacology. Pharmaceutical Biology. 2016;54(9):1901-1918.

72. Mekuria AB, Erku DA, Gebresillassie BM, et al. Prevalence and associated factors of herbal medicine use among pregnant women on antenatal care follow-up at University of Gondar referral and teaching hospital, Ethiopia: A cross-sectional study. BMC Complementary and Alternative Medicine. 2017;17(1):86.

73. Dugoua JJ. Herbal medicines and pregnancy. Journal of Population Therapeutics and Clinical Pharmacology. 2010;17(3):e370-e378.
74. Bruno LO, Simoes RS, de Jesus Simoes M, et al. Pregnancy and herbal medicines: An unnecessary risk for women's health-A narrative review. Phytotherapy Research. 2018;32:796-810.

75. Mate A. Medicinal and Aromatic Plants of the World. Scientifc, Production, Commercial and Utilization Aspects, vol. 1, Springer Science + Business Media: Dordrecht, Netherlands; 2015.

76. Terao J. Factors modulating bioavailability of quercetin related flavonoids and the consequences of their vascular function. Biochemical Pharmacology. 2017;139:15-23.

77. Romano B, Pagano E, Montanaro V, et al. Novel insights into the pharmacology of flavonoids. Phytotherapy Research. 2013;27(11):15881596.

78. Quinones M, Miguel M, Aleixandre A. Beneficial effects of polyphenols on cardiovascular disease. Pharmacological Research. 2013;68(1):125131.

79. Yamagata K, Tagami M, Yamori Y. Dietary polyphenols regulate endothelial function and prevent cardiovascular disease. Nutrition Journal. 2015;31(1):28-37.

80. Alves JLDB, de Sousa VP, Neto MPC, et al. New insights on the use of dietary polyphenols or probiotics for the management of arterial hypertension. Frontiers in Physiology. 2016;7:448.

81. Emerenciano V, Militao J, Campos C, et al. Flavonoids as chemotaxonomic markers for Asteraceae. Biochemical Systematics and Ecology. 2001;29(9):947-957.

82. Kumar S, Pandey AK. Chemistry and Biological Activities of Flavonoids: An Overview. The Scientifc World Journal. 2013;2013:162750.

83. Dixon RA, Pasinetti GM. Flavonoids and isofavonoids: from plant biology to agriculture and neuroscience. Plant Physiology. 2010;154(2):453-457.

84. Andersen M, Markham KR. Flavonoids, Chemistry, Biochemistry and Applications. CRC Press, Taylor \& Francis Group: Boca Raton, Fla, USA; 2006 .

85. Dharmashankar K, Widlansky ME. Vascular endothelial function and hypertension: insights and directions. Current Hypertension Reports. 2010;12(6):448-455.

86. Widmer RJ, Lerman A. Endothelial dysfunction and cardiovascular disease. Global Cardiology Science Practice. 2014;2014(3):291-308.

87. Konukoglu D, Uzun H. Endothelial dysfunction and hypertension Advances in Experimental Medicine and Biology. 2016;956:511-540.

88. Rastogi S, Pandey MM, Rawat AKS. Traditional herbs: a remedy for cardiovascular disorders. Phytomedicine. 2016;23(11): 1082-1089.

89. Kim Y, Je Y. Flavonoid intake and mortality from cardiovascular disease and all causes: A meta-analysis of prospective cohort studies. Clinical Nutrition ESPEN. 2017;20:68-77.

90. Chirumbolo S. Flavonoids in coronary heart disease. Trombosis Research. 2015;135(5):1040-1041.

91. Edwards RL, Lyon T, Litwin SE, et al. Quercetin reduces blood pressure in hypertensive subjects. Journal of Nutrition. 2007;137(11):2405-2411.

92. Bondonno NP, Bondonno CP, Hodgson JM, et al. The Efcacy of Quercetin in Cardiovascular Health. Current Nutrition Reports. 2015;4(4):290-303.

93. Gormaz JG, Quintremil S, Rodrigo R. Cardiovascular disease: A target for the pharmacological efects of quercetin. Current Topics in Medicinal Chemistry. 2015;15(17):1735-1742.

94. Todaka E, Sakurai K, Fukata H, et al. Fetal exposure to phytoestrogens - The difference in phytoestrogen status between mother and fetus. Environmental Research. 2005;99(2):195-203. 
95. Vanhees K, Godschalk RW, Sanders A, et al. Maternal quercetin intake during pregnancy results in an adapted iron homeostasis at adulthood. Toxicology. 2011;290(2-3):351-359.

96. Prater MR, Laudermilch CL, Liang C, et al. Placental Oxidative Stress Alters Expression of Murine Osteogenic Genes and Impairs Fetal Skeletal Formation. Placenta. 2008;29(9):802-808.

97. Liang C, Oest ME, Jones JC, et al. Gestational high saturated fat diet alters C57BL/6 mouse perinatal skeletal formation. Birth Defects Research Part $B$ - Developmental and Reproductive Toxicology. 2009;86(5):362-369.

98. Zhao YT, Wang XD, Shi WY, et al. Antiabortiveefect of quercetin and bornyl acetate on macrophages and IL-10 in uterus of mice. African Journal of Biotechnology. 2010;10(43):8675-8682.

99. Karampour NS, Arzi A, Varzi HN, et al. Quercetin preventive efects on theophyllineinduced anomalies in rat embryo. Jundishapur Journal of Natural Pharmaceutical Products. 2014;9(3):e17834.

100. Dogan Z, Elbe H, Taslidere E, et al. Efects of ciprofoxacin on fetal rat liver during pregnancy and protective efects of quercetin. Biotechnic \& Histochemistry. 2017;92(7):481-486.

101. Khaksary-Mahabady M, Ranjbar R, Najafzadeh-Varzi H, et al Protective efect of quercetin on histomorphometric changes in kidney of retinoid acid-treated rat fetuses. International Journal of Morphology. 2018;36(1):338-344

102. Toumi ML, Merzoug S, Tahraoui A. Efects of quercetin on predator stress-related hematological and behavioural alterations in pregnant rats and their offspring. Journal of Biosciences. 2016;41(2):237-249.
103. Huang JJ, Liu X, Wang XQ, et al. Efects of quercetin on the learning and memory ability of neonatal rats with hypoxic-ischemic brain damage. Zhongguo Dang Dai ErKe Za Zhi. 2012;146:454-457.

104. Liu W, Zhang M, Feng J, et al. The influence of quercetin on maternal immunity, oxidative stress, and infammation in mice with exposure of fne particulate matter during gestation. International Journal of Environmental Research and Public Health. 2017;14(592):1-16.

105. Braga CP, Baptista RD, Peixoto FB. Efects of Quercetin Administration on the Pregnancy Outcome of Diabetic Rats. Journal of Diabetes \& Metabolism. 2012;3(2):1-4.

106. Shahzad H, Giribabu N, Muniandy S, et al. Quercetin induces morphological and proliferative changes of rat's uteri under estrogen and progesterone influences. International Journal of Clinical and Experimental Pathology. 2014;7(9):5484-5494.

107. Xu L, Sun L, Lu L, et al. Efects of Quercetin on CYP450 and Cytokines in Aroclor 1254 Injured Endometrial Cells of the Pregnant Rats. BioMed Research International. 2014;2014:497508.

108. Lee M, Yun S, Lee H, et al. Quercetin Mitigates Infammatory Responses Induced by Vascular Endothelial Growth Factor in Mouse Retinal Photoreceptor Cells through Suppression of Nuclear Factor Kappa B. International Journal of Molecular Sciences. 2017;18(11):2497.

109. Ruchika LN, Sarika W. Recent advances in topical delivery of flavonoids A review. Journal of Controlled Release. 2019;296:190-201. 